\title{
EFEITOS DOS CONFLITOS DE USO DO SOLO EM ÁREAS DE PRESERVAÇÃO PERMANENTE SOBRE A PRODUÇÃO E TRANSPORTE DE SEDIMENTOS EM BACIAS HIDROGRÁFICAS: O CASO DA BACIA DO RIO DO COCO, TOCANTINS, BRASIL
}

\author{
EFFECTS OF SOIL USE CONFLICTS IN PERMANENT PRESERVATION AREAS ON THE \\ PRODUCTION AND TRANSPORT OF SEDIMENTS IN WATERSHEDS: CASE-STUDY OF THE \\ RIO DO COCO WATERSHED, TOCANTINS, BRAZIL
}

\author{
Rejane Freitas Benevides ALMEIDA ${ }^{1}$, Laerte Guimarães FERREIRA JUNIOR ${ }^{2}$, \\ Maximiliano BAYER ${ }^{2}$, José Luiz Cabral da SILVA JUNIOR ${ }^{3}$ \\ ${ }^{1}$ Instituto Federal de Tocantins. Campus Paraíso do Tocantins. Distrito Agroindustrial, BR 153, km 480, Paraíso do Tocantins - TO. \\ E-mail: rejane@ifto.edu.br \\ ${ }^{2}$ Universidade Federal do Goiás. Campus II Samambaia - Cx. Postal 131 - Goiânia, GO. \\ E-mail: laerte@ufg.br; maxibayer@yahoo.com.ar \\ ${ }^{3}$ Universidade Estadual do Tocantins. Q. 108 Sul Alameda 11, Lote 03 - Plano Diretor Sul, Palmas - TO. \\ E-mail: jlcabral_jr@yahoo.com.br

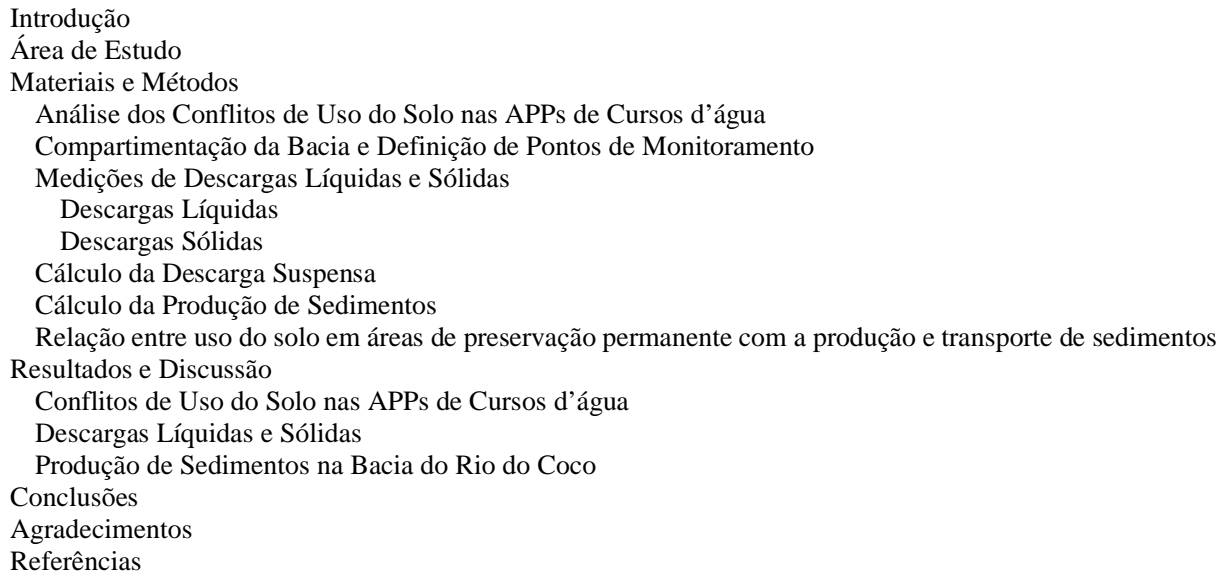

RESUMO - Objetivou-se avaliar os conflitos de uso do solo em áreas de preservação permanente e sua relação com a produção e transporte de sedimentos na bacia hidrográfica do Rio do Coco. Para tanto, foram realizadas medições de descargas líquidas e sólidas no exutório das principais sub-bacias que compõem a Bacia do Rio do Coco, bem como o mapeamento dos usos presentes nas APPs. As descargas sólidas foram analisadas por meio de coletas de amostras de água para a determinação dos sólidos em suspensão usandose a técnica de amostragem por integração vertical com uso de amostradores (DH-48 e DH-59). Já as medições de vazão foram realizadas através dos medidores de vazão ADV e ADCP. Para o mapeamento da cobertura e uso da terra nas APPs foram utilizadas imagens do satélite Landsat 8, ano 2015, sendo a segmentação das imagens realizada no ENVI, com posterior classificação por meio da inspeção visual. Os resultados indicaram que a região da alta bacia apresentou maiores irregularidades nas APPs, sendo também responsável pela maior produção e transporte de sedimentos, o que sugere uma relação com as atividades de uso na bacia.

Palavras-chave: Produção. Transporte. Sedimentos. Áreas de Preservação Permanente. Bacia do Rio do Coco.

ABSTRACT - Aimed at evaluate soil use conflicts in permanent preservation areas and their relationship with the production and transport of sediments in the Coco River basin. For that, were analized liquid and solid discharges carried out in the mouth of the main sub-basins, as well mapping of the uses in the PPA areas. The solid discharges were analyzed by collecting water samples to determine suspended solids using vertical integration sampling technique with samplers (DH-48 and DH-59). Flow measurements were performed through flow meters ADV and ADCP. For the mapping of land use and coverage in PPAs, were used images the Landsat 8 satellite, year 2015, being the segmentation of the images realized in the ENVI, with subsequent classification through visual inspection. The results indicated that the region of the high basin presented more irregularities in the PPAs, being also responsible for the higher production and transport of sediments, which suggests a relation with the use activities in the basin.

Keywords: Production. Transport. Sediments. Permanent Preservation Areas. Basin of the Coco River.

\section{INTRODUÇÃO}

A dinâmica de transporte de sedimentos e (Suaréz et al., 2016), somados a estes fatores, solutos é determinada por um conjunto complexo Panwar et al. (2016) citam que a perturbação de variáveis como litologia, clima e topografia antrópica pode controlar significativamente a 
variação espacial e temporal da carga de sedimentos nos rios, afetando toda a bacia hidrográfica, uma vez que esta é um sistema aberto, onde o fluxo de matéria e energia é dinâmico e altamente dependente da gestão dos solos (Zanata et al., 2015). González \& Jaramillo (2010) complementam ainda que os sedimentos constituem uma interseção entre os recursos do solo e os recursos hídricos e que as mudanças nos padrões de sedimentos podem ser reflexos das alterações no uso do solo de uma bacia e aproveitamento da mesma.

Dentre as principais causas das perdas de solo em bacias hidrográficas, destaca-se a mobilização de sedimentos causados pela erosão de terras, fortemente ligada à mudança no uso do solo (Hartwig, et al., 2016). Costa et al. (2016) destacam como principais contribuintes para as perdas de solos, o manejo inadequado por meio de práticas agrícolas intensivas e a falta de manejo em áreas cultivadas com pastagens e o desmatamento.

Antoneli (2011) cita que dentro desse processo, a vegetação tem um papel de fundamental importância, sendo a diminuição do escoamento superficial uma de suas principais funções hidrológicas (Rodrigues et al., 2015). Esta atua como um regulador de entrada e permanência de água no solo, sendo a sua remoção, um potencializador para os efeitos pluvioerosivos, uma vez que o solo fica exposto.

Hartwig et al. (2016) descrevem que o problema torna-se mais agravante quando a degradação ocorre nas áreas de mata ciliar, pois a estabilidade das margens dos rios tende a se tornar enfraquecidas, potencializando a entrada de sedimentos para o corpo hídrico, principalmente, quando a área apresenta naturalmente características topográficas e propriedades dos solos que favorecem as perdas por erosão.

Santos \& Sparovek (2011), ao avaliarem a eficiência da mata ciliar na retenção dos sedimentos gerados em área agrícola cultivada com algodão, observaram a presença de depósitos de sedimentos na área de mata ciliar se estendendo por mais de 20 metros na mata, confirmando a importância desta na contenção dos sedimentos e na proteção e manutenção da qualidade e quantidade de água presente nas bacias.

Portanto, estudar os conflitos de uso em APP e sua relação com a produção e transporte de sedimentos em bacias hidrográficas torna-se de suma relevância, uma vez que os resultados podem ser determinantes no processo da gestão adequada dos recursos hídricos.

Deste modo, o presente trabalho teve como objetivo analisar os conflitos de uso do solo nas áreas de preservação permanente e sua relação com a produção e o transporte de sedimentos na bacia do Rio do Coco e de seus principais rios constituintes, identificando as áreas com maiores contribuições para o Rio do Coco e o papel das mesmas dentro do processo hidrossedimentológico na bacia.

\section{ÁREA DE ESTUDO}

A bacia do Rio do Coco localiza-se na margem direita da bacia hidrográfica do Rio Araguaia, no estado do Tocantins, entre os municípios de Caseara, Marianópolis do Tocantins, Divinópolis do Tocantins, Monte Santo, Paraíso do Tocantins, Chapada de Areia, Pium e Barrolândia. Esta bacia é classificada como de sétima ordem, de acordo com Strahler (1952) e ocupa uma área aproximada de $6.670 \mathrm{Km}^{2}$. O principal curso d'água na bacia é o Rio do Coco, o qual possui um comprimento de aproximadamente 356 quilometros. As nascentes principais estão localizadas no município de Paraíso do Tocantins, nas proximidades da Serra do Estrondo e sua foz localiza-se na cidade de Caseara. Além do Rio do Coco, a bacia possui outros cursos d'água de grande relevância, dentre os quais destacam-se: o Ribeirão Piedade, o Ribeirão Surubim e o Ribeirão Prata (Figura 1).

\section{MATERIAIS E MÉTODOS}

Os procedimentos metodológicos para o desenvolvimento do trabalho foram divididos em etapas, sendo: análise dos conflitos de uso do solo nas áreas de preservação permanente de cursos d’água; compartimentação e definição dos pontos de monitoramento; medição das descargas líquidas e sólidas; cálculo da descarga total e cálculo da produção de sedimentos.

\section{Análise dos Conflitos de Uso do Solo nas APPs de Cursos d’água}

Para análise dos conflitos de uso do solo nas áreas de preservação permanente foram elaborados mapas de cobertura e uso da terra para as APPs de curso d'água de cada sub-bacia.

Inicialmente realizou-se a vetorização manual da rede de drenagem na escala 1:5.000, a partir 
de imagens do satélite RapidEye (2011), sendo as nascentes obtidas por meio da marcação de pontos nas extremidades de todos os canais de ordem 1, definidos por meio da hierarquização da rede de drenagem, conforme classificação de Strahler (1952). Já os lagos, as lagoas e as represas foram extraídas da classificação de imagens, onde foram considerados apenas os polígonos acima de um hectare.

A partir da rede de hidrográfica deu-se início ao processamento dos dados, sendo criados buffers de APPs, de acordo com os parâmetros estabelecidos de proteção na legislação ambiental (Lei 12.651/2012).

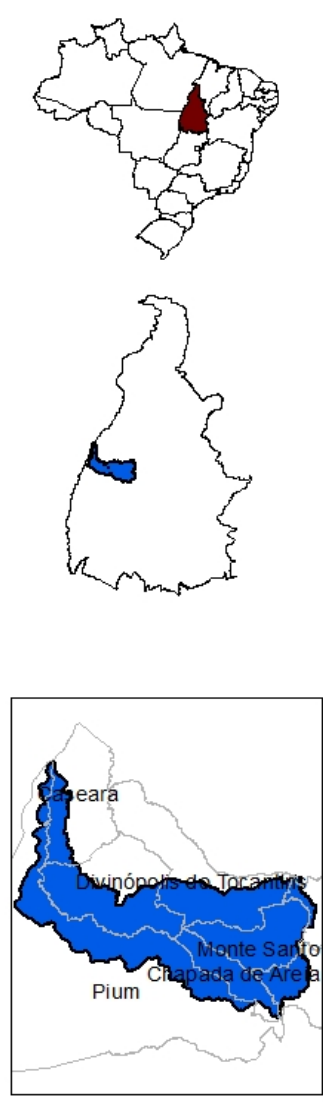

LOCALIZAÇÃO DA BACIA DO RIO DO COCO
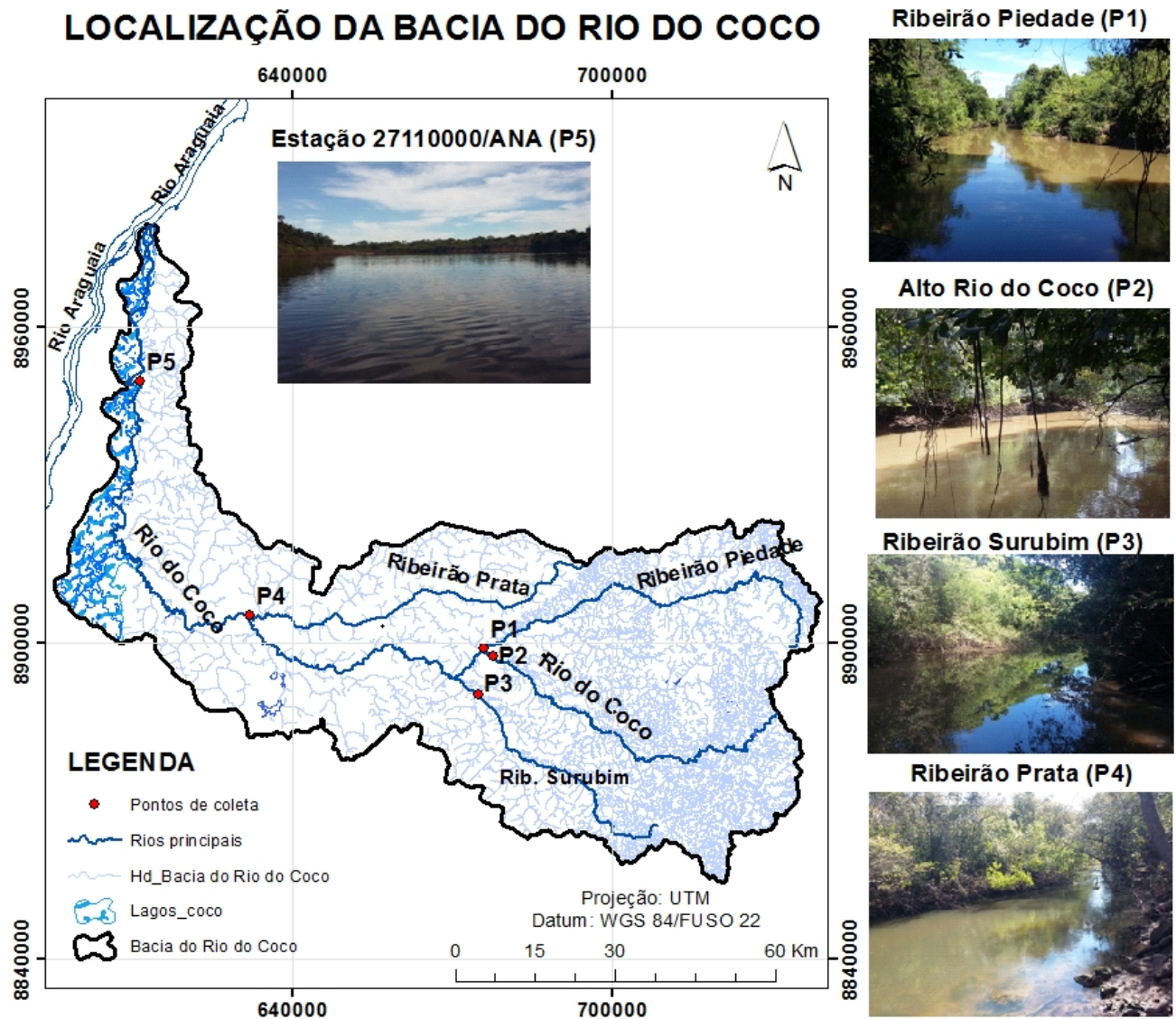

Ribeirão Prata (P4)

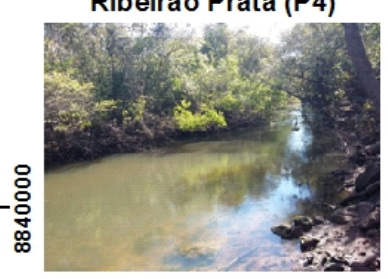

Figura 1 - Localização da Bacia do Rio do Coco, com destaque para o posicionamento dos pontos de monitoramento.

Para o mapeamento da cobertura e uso da terra nas APPs foram utilizadas imagens do satélite Landsat 8 (sensor OLI - Operational Land Imager), cenas 222/67 e 223/67 (órbita/ponto), ano 2015, as quais foram registradas para o fuso UTM 22 S, Datum WGS 1984, sendo a segmentação da imagem realizada no ENVI a partir da ferramenta Feature extraction. Os parâmetros adotados foram: segmentação pelo algoritmo Edge com nível de escala 60 e merge pelo algoritmo Full Lambda Schedule com nível 90 e textura tipo Kernel, tamanho 3.

Para melhorar a distinção entre as classes, o procedimento de classificação considerou imagens dos períodos seco (agosto) e chuvoso (abril), sendo a classificação dos segmentos realizada por meio da inspeção visual, utilizando uma escala de visualização (zoom) de 1:20:000. Tal análise teve como apoio as imagens provenientes da plataforma Google Earth e imagens RapidEye de 2013 (resolução espacial de $5 \mathrm{~m}$ ), além de comparações com a base de dados dos mapeamentos de uso da terra e cobertura vegetal remanescente do Estado do Tocantins (2007) e Projeto TerraClass Cerrado (2013).

Visando analisar a concordância entre o mapa classificado e a realidade local, foram coletados 272 pontos ao longo de toda a bacia com GPS de navegação durante duas campanhas de campo executadas nos meses de junho e agosto de 2016. Os pontos coletados, posteriormente, foram utilizados no cálculo da exatidão global a partir de uma matriz de confusão, obtendo-se, dessa forma, uma estimativa de exatidão da classificação considerada satisfatória (92,27\%).

Os mapas de cobertura e uso da terra para as APPs foram obtidos por meio da sobreposição do mapa gerado com a classificação da imagem e os buffers de APPs para cada bacia. Após esta sobreposição, foram mensuradas as áreas 
correspondentes a cada classe, assim como os percentuais de uso em APPs a partir de cálculos de área.

\section{Compartimentação da Bacia e Definição de Pontos de Monitoramento}

A compartimentação da bacia do Rio do Coco foi realizada com base no perfil longitudinal do rio principal (Rio do Coco), sendo possível identificar dois compartimentos (alta e baixa bacia), para os quais definiram-se sub-bacias principais, utilizando o critério de ordens, conforme classificação de Sthraler (1952). Na região da alta bacia ficaram as sub-bacias de $6^{\circ}$ ordem: Alto Rio do Coco, Ribeirão Piedade e Ribeirão Surubim, já na região da baixa bacia foram incluídas as sub-bacias do Ribeirão Prata ( $5^{\circ}$ ordem) e Baixo Rio do Coco ( $7^{\circ}$ ordem).

Para cada sub-bacia foi identificada o canal de drenagem principal, para as quais foram definidas seções de medição, onde foram realizadas medições de descargas líquidas e sólidas.

É importante mencionar que a seção de medição da sub-bacia do Baixo Rio do Coco está localizada em um trecho onde já recebeu toda a drenagem das sub-bacias analisadas neste estudo (Alto Rio do Coco, Ribeirão Piedade, Ribeirão Surubim e Ribeirão Prata), sendo considerado o exutório da grande bacia do Rio do Coco. Além disso, o local escolhido está localizado no ponto de monitoramento hidrológico da Agência Nacional de Águas (ANA) (Estação 27110000). A escolha do mesmo foi intencional, uma vez que é o único ponto na bacia que possui um monitoramento sistemático. As medições foram iniciadas em abril de 2015, estendendo-se até abril de 2016, a partir de medições bimestrais.

Antes do início das medições foram realizadas visitas nos pontos previamente selecionados para o reconhecimento e definição das seções de medição. Priorizou-se trechos retilíneos, sendo evitados locais perto de confluências (desembocadura de outros canais). Cada seção foi identificada por coordenadas em UTM além de marcações em estruturas (rochas e árvores) que pudessem facilitar o acesso durante o período de monitoramento.

Menciona-se ainda que a escolha dos pontos de monitoramento buscou atender ao critério de maior proximidade com a foz das bacias analisadas, pois pretendia-se avaliar a contribuição de sedimentos de cada canal para o Rio do Coco.

\section{Medições das Descargas Líquidas e Sólidas Descargas líquidas}

As seções analisadas apresentaram características bem distintas entre si, possuindo os canais de drenagem uma variação significativa em função da sazonalidade. Observaram-se tantas as situações de transbordamento da calha dos rios no período chuvoso, como a diminuição considerável do volume de água, chegando até mesmo a condições de intermitência temporária em alguns canais durante o período seco. Diante das especificidades encontradas, as medições de vazão foram realizadas obedecendo às peculiaridades apresentadas pelos canais em cada período analisado, o que exigiu um intenso esforço de campo com logística e equipamentos apropriados.

Para os cursos d’água com profundidade inferior a 1,5 metros, a vazão foi obtida por meio do medidor de vazão ADV (Acoustic Doppler Velocimeter) FlowTracker. Já para as situações em que o canal de drenagem possuía profundidades superiores, as medições de vazão foram realizadas por meio da utilização do ADCP (Acoustic Doppler Current Profiler).

\section{Descargas sólidas}

As descargas sólidas foram analisadas por meio de coletas de amostras de água para a determinação dos sólidos em suspensão usandose a técnica de amostragem por integração vertical com uso de amostradores (DH-48 e DH59), de acordo com a metodologia descrita por Carvalho (1994).

Após coletadas, as amostras foram acondicionadas e mantidas refrigeradas até serem encaminhadas ao Laboratório de Saneamento do Instituto Federal do Tocantins/Campus Paraíso onde foi executada a determinação da concentração dos sólidos em suspensão. Esta seguiu a metodologia proposta por Carvalho (2000b), sendo realizada a filtragem de um volume de amostra conhecido utilizando-se uma bomba de sucção a vácuo e filtros em microfibra de vidro, com porosidade de $47 \mu \mathrm{m}$ de diâmetro. A concentração de sólidos totais $(\mathrm{mg} / \mathrm{L})$ é o resultado da divisão da massa retida no filtro seco (em estufa $100-110^{\circ} \mathrm{C}$ durante 1 hora), pelo volume de amostra filtrada.

A pesagem dos filtros foi realizada em balança de precisão, sendo os valores medidos em gramas (g). A concentração dos sólidos suspensos totais foi obtida a partir da seguinte fórmula: 


$$
C S S=\frac{(P 1-P 0) \times 1000000}{V A}
$$

Onde:

Css = Concentração de sólidos suspensos totais (mg/L);

$\mathrm{P}_{0}=$ Peso inicial do filtro em (g);

$\mathrm{P}_{1}=$ Peso apresentado pelo filtro após a secagem em estufa (g)

$\mathrm{VA}=$ Volume da amostra (L)

\section{Cálculo da Descarga Suspensa}

Para a determinação da descarga suspensa de sedimentos adotou-se o cálculo do transporte da descarga pelo método simplificado de Colby (1957, in Carvalho, 2000a), onde são necessários os valores da descarga líquida (Q), profundidade média (p), velocidade média da corrente (v), largura da seção (L) e concentração de sólidos em suspensão (Css). A descarga suspensa é obtida conforme a equação abaixo:

$$
Q s S=0,0864 x Q x C s S
$$

Onde:

Qss - descarga em suspensão em ton/dia;

$\mathrm{Q}$ - descarga líquida em $\mathrm{m}^{3} / \mathrm{s}$;

Css - concentração média em mg/L.

\section{Cálculo da Produção de Sedimentos}

A produção de sedimentos em suspensão (Pss) foi obtida pela equação 03 abaixo, conforme Carvalho (2008):

\section{RESULTADOS E DISCUSSÃO}

\section{Conflitos de Uso do Solo nas APPs de Cursos d'água}

A figura 2 apresenta os percentuais de áreas com conflitos de uso em áreas de preservação permanente na bacia do Rio do Coco e suas subbacias.

Como pode ser observado, a bacia do Rio do

$$
P S S=\frac{Q s S}{A}
$$

Onde:

Pss $=$ produção de sedimentos em suspensão (ton. $\mathrm{km}^{-2}$. $\mathrm{mês}^{-1}$ )

Qss = descarga sólida de sedimentos em suspensão (ton. mês ${ }^{-1}$ )

A = área incremental da sub-bacia $\left(\mathrm{km}^{2}\right)$

Relação Entre Uso do Solo em Áreas de Preservação Permanente com a Produção e Transporte de Sedimentos

Para avaliar os efeitos do uso do solo na produção e transporte de sedimentos realizou-se uma análise dos conflitos de uso do solo em APPs para cada sub-bacia.

Tal análise permitiu identificar as áreas com maiores irregularidades em APPs, revelando o contexto de degradação de cada região.

Posteriormente, os dados dos conflitos de uso do solo em APPs foram comparados com os resultados de produção e transporte de sedimentos para cada sub-bacia, sendo possível identificar as áreas com maior potencial para a produção e transporte de sedimentos na grande Bacia do Rio do Coco e o papel das mesmas dentro do processo hidrossedimentológico.

Coco e suas sub-bacias apresentam percentuais diferenciados de conflitos de uso ao longo das APPs.

Quando se considera toda a área da bacia, observa-se que 32,8\% das APPs encontram-se algum tipo de uso, sendo destes 32,37\% representados pelos conflitos com a pastagem.

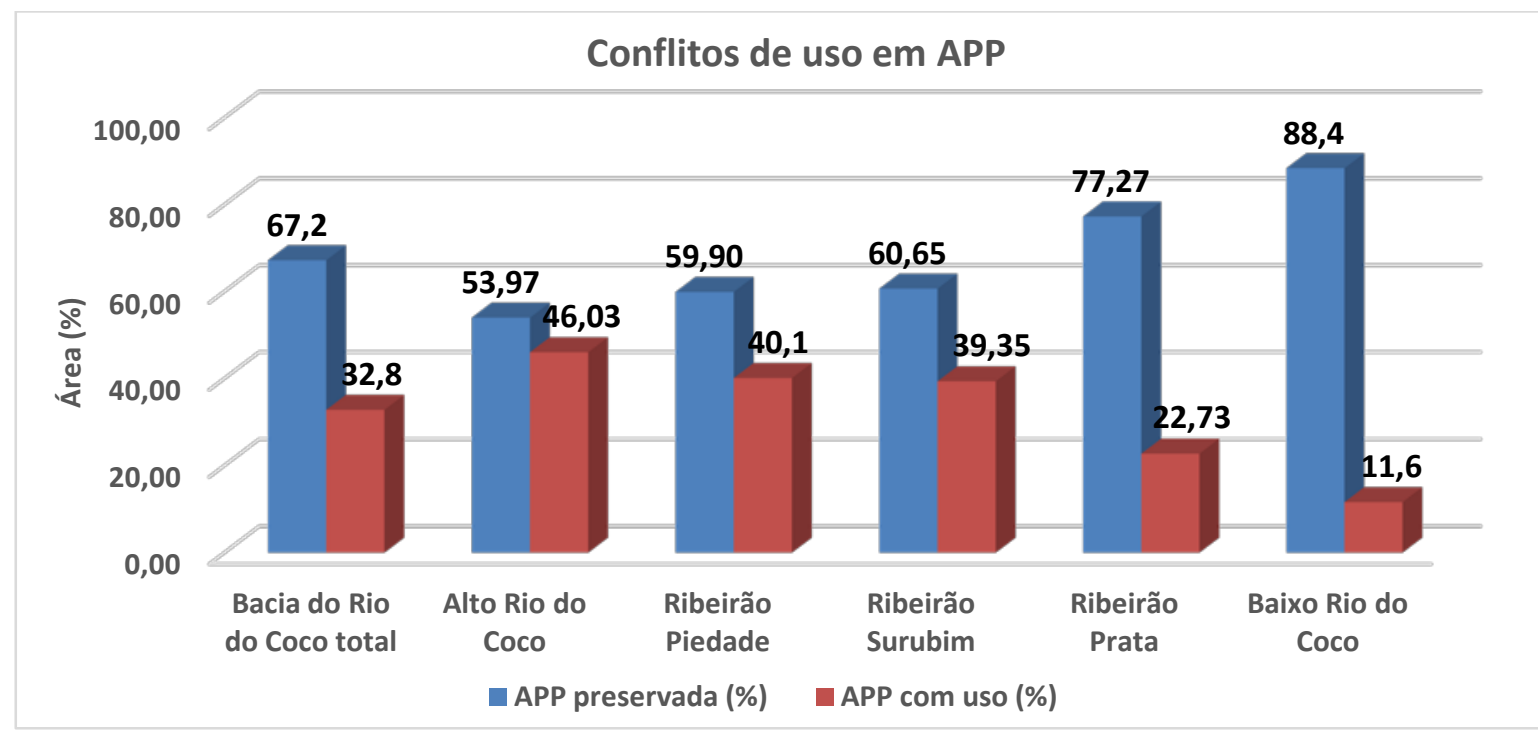

Figura 2 - Conflitos de uso em APPs nos compartimentos da bacia do Rio do Coco. 
Resultados similares foram encontrados por Silva et al. (2016) na bacia do Ribeirão dos Pinheirinhos em Brotas (SP), onde observaramse 32,09\% das APPs ocupadas com alguma atividade irregular, principalmente pela pastagem.

Ao se analisar individualmente cada subbacia, conclui-se que os conflitos de uso da terra atingem percentuais variados, chegando até 46,03\%, conforme apresentado para o Alto Rio do Coco, a qual apresentou maior percentual de uso em APP, seguido pelas sub-bacias dos Ribeirões Piedade e Surubim com 40,1\% e 39,35\%, consecutivamente.

No que diz respeito às demais áreas analisadas, a sub-bacia do Baixo Rio do Coco foi a que apresentou menor conflito em APP, com $11,6 \%$, seguido pela sub-bacia do Ribeirão Prata com 22,73\%. Quando se comparam as sub-bacias pertencentes ao compartimento da alta bacia com o grupo de sub-bacias da região da baixa bacia do Rio do Coco observa-se que as irregularidades estão mais presentes nas sub-bacias da alta bacia. Vale ressaltar que, nestas áreas, o processo de uso e ocupação das terras se deu de forma mais intensa, influenciado, principalmente, pela atividade agropecuária. Por outro lado, é interessante destacar que o sistema de drenagem das bacias analisadas pode também ter influenciado nos resultados. A alta bacia possui maior número de canais por área e, consequentemente necessita de mais áreas destinadas a APP, conforme demonstrado na figura 3.

Após análise da figura 3, pode-se observar que as sub-bacias da alta bacia do Rio do Coco (Alto Rio do Coco, Ribeirão Piedade e Ribeirão Surubim) possuem uma densidade de drenagem mais desenvolvida em relação às sub-bacias da baixa bacia do Rio do Coco (Baixo Rio do Coco e Ribeirão Prata).

Essa assertiva pode ser confirmada pelos resultados obtidos por Almeida et al. (2016) em estudos morfométricos realizados na bacia hidrográfica do Rio do Coco, a qual foi subdividida, quanto a sua drenagem em: a) bem drenada - sub-bacia do Alto Rio Coco (2,104 $\mathrm{km} / \mathrm{km}^{2}$ ); b) drenagem média - sub-bacias do Ribeirão Piedade $\left(1,55 \mathrm{~km} / \mathrm{km}^{2}\right)$ e Ribeirão Surubim $\left(1,321 \mathrm{~km} / \mathrm{km}^{2}\right)$ e c) drenagem pobre sub-bacias do Ribeirão Prata $\left(0,62 \mathrm{~km} / \mathrm{km}^{2}\right)$ e Baixo Rio Coco $\left(0,636 \mathrm{~km} / \mathrm{km}^{2}\right)$.

É importante mencionar que a presença de maiores irregularidades em APPs na região da alta bacia pode influenciar diretamente na quantidade de material produzido pela bacia e transportado pelo canais. Haja vista que a vegetação tem um papel fundamental para a estabilidade dos solos e, com consequente minimização dos efeitos dos processos pluvioerosivos.

Desse modo, analisa-se que os conflitos de uso podem ter sido determinantes nos resultados desta pesquisa, no entanto, não se pode desconsiderar os aspectos físicos de cada subbacias, como já citado acima, a região que compreende a alta bacia possui características de drenagem e relevo que podem ser importantes em análises como estas.

\section{Descargas Líquidas e Sólidas}

A tabela 1 apresenta os resultados das descargas líquidas e sólidas encontradas para os pontos de monitoramento analisados. Como pode ser observado, os resultados apresentam uma relação direta com a precipitação.

Observa-se que a diminuição dos índices pluviométricos tem efeitos diretos sobre a disponibilidade de água nos canais de drenagem avaliados. A exemplo disso, citam-se os Ribeirões Surubim e Prata, que apresentaram uma variação considerável entre as estações seca e chuvosa, sendo observado, em alguns meses de estiagem, a interrupção de seus fluxos.

As sub-bacias com maiores contribuições em volume de água para o Rio do Coco são representadas pelo Ribeirão Piedade e Alto Rio do Coco, sendo os Ribeirões Prata e Surubim responsáveis pelas menores parcelas de vazão, concordando com os resultados de transporte de sedimentos observados para essas sub-bacias.

No Baixo Rio do Coco/Estação ANA 27110000 foi registrado uma grande variação nos resultados de vazão, tendo o menor valor observado no mês de outubro com 1,1304 m³/s e maior valor no mês de fevereiro de 2016 com $208,9 \mathrm{~m}^{3} / \mathrm{s}$. Essa grande oscilação no volume de água para este ponto também pode ser resultante da conectividade do Rio do Coco com alguns lagos que compõem a região do Cantão no período chuvoso. Ressalta-se ainda que em várias amostragens, o volume de todos os quatro afluentes não representou mais do que 30 a $35 \%$ da vazão registrada neste ponto, evidenciando a grande influência dos lagos e do próprio Araguaia no incremento da vazão durante as cheias. 


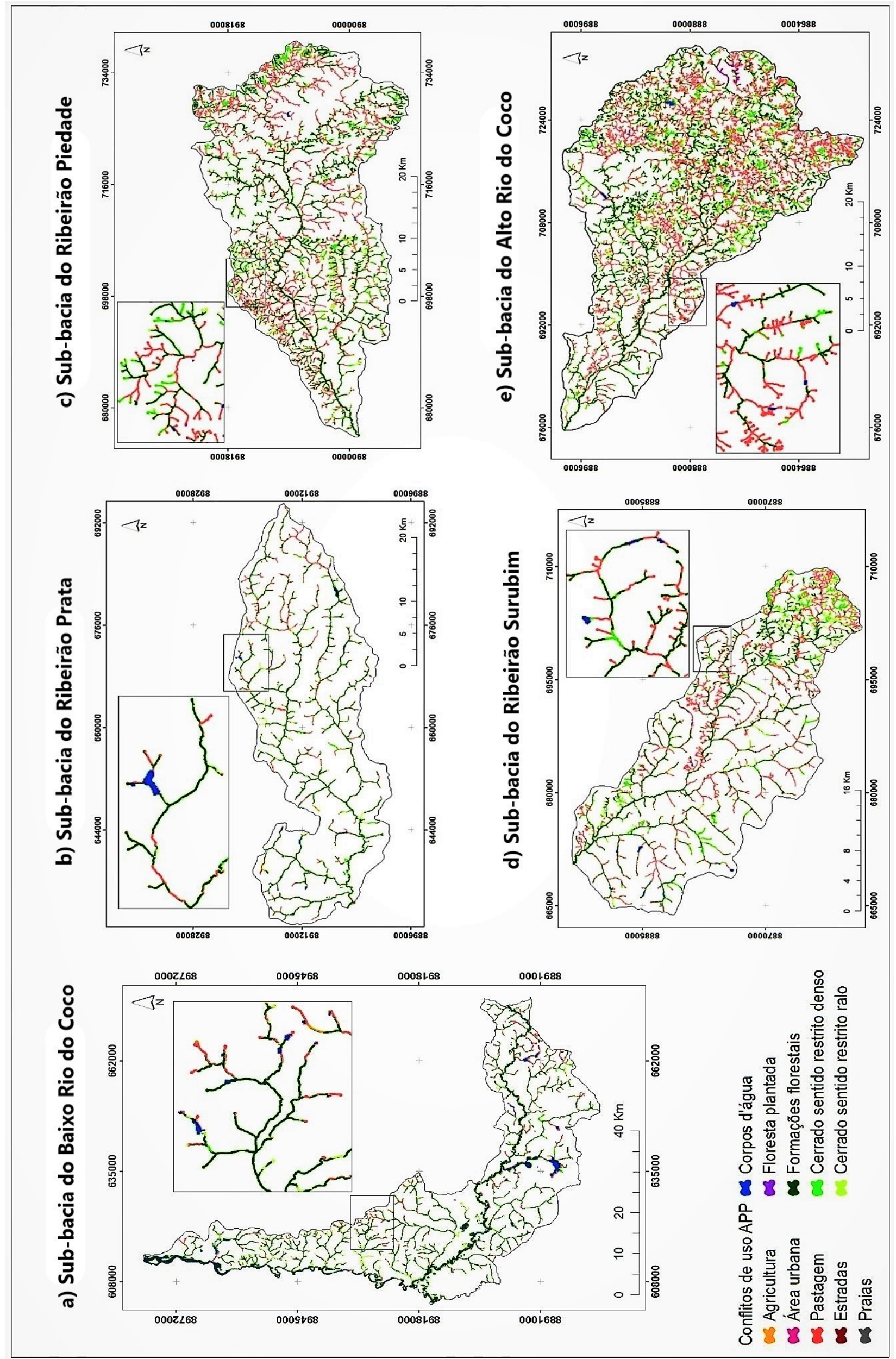

Figura 3 - Conflitos de uso do solo nas áreas de preservação permanente nas sub-bacias do Rio do Coco. 
Tabela 1 - Descargas líquidas e sólidas nas sub-bacias principais da bacia do Rio do Coco.

\begin{tabular}{|c|c|c|c|c|c|c|c|c|c|c|c|c|c|c|c|c|}
\hline \multirow{3}{*}{ Ano } & \multirow{3}{*}{ is } & \multicolumn{3}{|c|}{ Ribeirão Piedade } & \multicolumn{3}{|c|}{ Alto Rio do Coco } & \multicolumn{3}{|c|}{$\begin{array}{l}\text { Ribeirão } \\
\text { Surubim } \\
\end{array}$} & \multicolumn{3}{|c|}{ Ribeirão Prata } & \multicolumn{3}{|c|}{$\begin{array}{c}\text { Baixo Rio do } \\
\text { Coco/Est. ANA }\end{array}$} \\
\hline & & $\mathbf{Q}$ & Css & Qss & $\mathbf{Q}$ & Css & Qss & $\mathbf{Q}$ & Css & Qss & $\mathbf{Q}$ & Css & Qss & $\mathbf{Q}$ & Css & Qss \\
\hline & & $\stackrel{\infty}{m}$ & 定 & 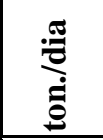 & $\stackrel{\infty}{m}$ & 它 & 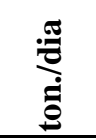 & $\stackrel{\infty}{m}$ & $\underset{\Xi}{\stackrel{1}{J}}$ & 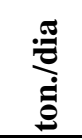 & $\stackrel{\infty}{m}$ & 它 & 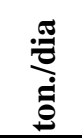 & $\stackrel{\infty}{m}$ & 兽 & 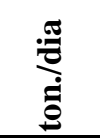 \\
\hline \multirow{5}{*}{2015} & Abr & 15,8 & 3 & 4,1 & 23,2 & 4 & 8,01 & - & 1 & - & 10,3 & 2 & 1,77 & - & 1 & - \\
\hline & Jun & 9,3 & 3 & 25,7 & 10,2 & 27 & 23,79 & 1,5 & 10 & 1,30 & 5,9 & 2 & 1,03 & 106,4 & 10 & 91,96 \\
\hline & Ago & 1,02 & 16,8 & 1,47 & 0,27 & 6 & 0,14 & $\mathrm{~S}$ & $\mathrm{~S}$ & $\mathrm{~S}$ & 0,45 & 4 & 0,15 & 4,72 & 7,6 & 3,10 \\
\hline & Out & 0,68 & 21,2 & 1,24 & S & $S$ & $\mathrm{~S}$ & $S$ & $\mathrm{~S}$ & $S$ & $\mathrm{~S}$ & $S$ & S & 1,13 & 4,8 & 0,47 \\
\hline & Dez & 3,77 & 22 & 7,16 & 3,01 & 40 & 10,39 & $\mathrm{~S}$ & $S$ & $\mathrm{~S}$ & $\mathrm{~S}$ & $\mathrm{~S}$ & S & 1,64 & 19,2 & 2,72 \\
\hline \multirow{2}{*}{2016} & $\mathrm{Fe}$ & 21,4 & 8 & 14,81 & 16,3 & 14 & 19,66 & 15,5 & 5,2 & 6,98 & 10,1 & 3,6 & 3,15 & 208,9 & 5,6 & 101,0 \\
\hline & Abr & 13,8 & 8 & 105,0 & 7,35 & 80 & 50,8 & 4,93 & 36 & 15,3 & 8,23 & 13 & 9,24 & 105,1 & 21 & 190,6 \\
\hline \multicolumn{2}{|c|}{ Média } & 9,4 & 27,3 & 22,78 & 10,04 & 28,5 & 18,8 & 7,32 & 13,05 & 7,87 & 7,00 & 4,9 & 3,07 & 71,32 & 9,98 & 65,00 \\
\hline
\end{tabular}

Nota: Q = vazão (m3/s); Css = concentração de sedimentos em suspensão (mg/L); Qss = descarga sólida em suspensão (ton./dia); S = curso d'água seco.

No que diz respeito à descarga sólida em suspensão, o quadro também sugere relação direta com a sazonalidade. Os meses que compreendem o período seco apresentaram os menores índices de descarga em relação aos demais meses avaliados, resultado das menores concentrações de sólidos em suspensão e vazão. Minela \& Merten (2011) descrevem que durante a cheia de uma bacia ocorre a maior parte da produção de sedimentos, sendo no momento das chuvas transportadas muitas partículas para o rio. Entretanto, após certo tempo, quando curso d’água atinge um volume considerável (vazão máxima), o carreamento tende a uma diminuição substancial. Em todas as sub-bacias foram observados esse comportamento, observando-se para no mês de abril de 2015 os menores valores registrados para a concentração de sedimentos em suspensão, o que pressupõe que o pico de transporte de sedimentos ocorre nos meses iniciais e finais do período chuvoso, estando adiantado do pico de vazão máxima.

Salienta-se que as sub-bacias da alta bacia apresentaram as maiores descargas em relação as demais sub-bacias analisadas, com destaque para o Ribeirão Piedade e o Alto Rio do Coco que no mês de abril de 2016 lançaram no Rio do Coco uma descarga sólida em suspensão de 105 e 50,80 ton./dia. Tais resultados indicam que as áreas com maiores irregularidades em APPs estão transportando volumes de sedimentos superiores às regiões com menores irregularidades, o que sugere uma relação direta entre os conflitos de uso em APP com os materiais transportados pelos canais.

As sub-bacias dos Ribeirões Surubim e Prata apresentaram as menores descargas para o Rio do Coco em relação aos demais canais avaliados, sobretudo o Ribeirão Prata, o qual se destacou com os resultados mais baixos, o que pode ser justificado pelos baixos resultados de sólidos em suspensão e vazão.

No que diz respeito ao Baixo Rio do Coco/Estação ANA 27110000, verificaram-se descargas mais elevadas nos meses de fevereiro e abril de 2016, onde foram registrados 101,07 e 190,68 ton/dia, respectivamente. Ressalta-se que este ponto se diferencia dos demais, sendo considerado o exutório da bacia do Rio do Coco, assim, os resultados apresentados indicam as descargas lançadas por esta bacia no Rio Araguaia.

Na tabela 2 estão representados os dados de monitoramento hidrossedimentológico realizado pela ANA no Rio do Coco/Estação 27110000 no período de 2008 a 2015. Como pode ser observado na tabela 2 , os dados coletados tiveram uma variação significativa em função da sazonalidade ao longo dos oito anos de monitoramento de descarga de sedimentos na estação 27110000 pela ANA, sendo registrado os maiores valores de descarga nos meses chuvosos.

A vazão média calculada para a estação foi de $120,43 \mathrm{~m}^{3} / \mathrm{s}$. Já as vazões máxima e mínima foram: 263,66 m³ $/ \mathrm{s}$ (fevereiro de 2012) e 0,99 $\mathrm{m}^{3} / \mathrm{s}$ (outubro de 2015), respectivamente. Notase que existe uma oscilação significativa nos resultados de vazão no ponto monitorado pela 
ANA, sugerindo grande interferência do ambiente de planície durante a estação chuvosa. Tal situação coloca a escolha da localização da estação (27110000) em questionamento, uma vez que esta pode não representar a real vazão produzida pelo Rio do Coco.

Tabela 2 - Descarga sólida em suspensão do Rio do Coco/Estação ANA 27110000.

\begin{tabular}{|c|c|c|c|c|c|c|c|}
\hline Ano & $\begin{array}{c}\text { Data } \\
\text { medição }\end{array}$ & Cota $(\mathbf{c m})$ & $\begin{array}{l}\text { Vazão } \\
\left(\mathrm{m}^{3} / \mathrm{s}\right)\end{array}$ & $\begin{array}{l}\text { Largura } \\
\text { (m) }\end{array}$ & $\begin{array}{l}\text { Vel. média } \\
(\mathrm{m} / \mathrm{s})\end{array}$ & SST (ppm) & $\begin{array}{c}\text { Descarga sólida } \\
\text { (ton./dia) }\end{array}$ \\
\hline \multirow{2}{*}{2008} & $04 / 2008$ & 538 & 245 & 115 & 0,47 & 2,75 & 58,21 \\
\hline & 08/2008 & 53 & 7,8 & 72,5 & 0,255 & 7,51 & 5,09 \\
\hline \multirow{2}{*}{2009} & $05 / 2009$ & 480 & 282 & 115 & 0,627 & 4,495 & 109,5 \\
\hline & 09/2009 & 50 & 8,2 & 65 & 0,295 & 8,776 & 6,27 \\
\hline \multirow{2}{*}{2010} & $03 / 2010$ & 491 & 254 & 115,8 & 0,549 & 6,235 & 136,8 \\
\hline & 07/2010 & 75 & 15,1 & 84,6 & 0,322 & 16,89 & 22,03 \\
\hline 2011 & $07 / 2011$ & 79 & 15,2 & 75 & 0,34 & 8,43 & 11,07 \\
\hline \multirow{3}{*}{2012} & $02 / 2012$ & 667 & 263,6 & 114,4 & 0,428 & 164,1 & $3.738,2$ \\
\hline & 06/2012 & 180 & 62,6 & 92 & 0,466 & 9,3 & 50,35 \\
\hline & $11 / 2012$ & 115 & 27,8 & 87,5 & 0,51 & 20,2 & 48,59 \\
\hline \multirow{2}{*}{2013} & $03 / 2013$ & 548 & 228,4 & 114 & 0,453 & 2,92 & 57,64 \\
\hline & $07 / 2013$ & 120 & 28,3 & 88 & 0,364 & 10,2 & 24,96 \\
\hline \multirow{2}{*}{2014} & $03 / 2014$ & 646 & 227,7 & 115,5 & 0,365 & 48,9 & 962,0 \\
\hline & $07 / 2014$ & 73 & 12,01 & 66,9 & 0,305 & 11,6 & 12,03 \\
\hline \multirow{2}{*}{2015} & $03 / 2015$ & 471 & 247,7 & 112,01 & 0,632 & 4,6 & 98,46 \\
\hline & 10/2015 & 21 & 0,99 & 26,8 & 0,124 & 14,1 & 1,206 \\
\hline \multicolumn{2}{|c|}{ Média } & 287,9 & 120,4 & 91,25 & 0,41 & 21,31 & 333,91 \\
\hline \multicolumn{2}{|c|}{ Máxima } & 667 & 263,6 & 115,8 & 0,632 & 164,1 & $3.738,2$ \\
\hline \multicolumn{2}{|c|}{ Mínima } & 21 & 0,99 & 26,8 & 0,124 & 2,75 & 1,206 \\
\hline
\end{tabular}

Fonte: Sistema de Informações Hidrológicas - HidroWeb/ANA.

A concentração de sedimentos em suspensão apresentou uma média de $21,31 \mathrm{mg} / \mathrm{L}$, tendo a mínima de 2,75 mg/L em abril de 2008 e máxima de 164,01 mg/L em fevereiro de 2012.

Em relação à descarga sólida de sedimentos em suspensão o valor médio encontrado foi de 333,91 ton/dia, variando de 1,206 ton/dia (valor mínimo) no mês de outubro de 2015 e 3.738,27 ton./dia no mês de fevereiro de 2012 (valor máximo).

O mês de março de 2014 também merece destaque, pois a descarga sólida chegou a 962,01 ton./dia. Essa variação no transporte de sedimentos também foi observada por Garrido et al. (2014) na bacia do Rio Piancó-Piranhas-Açu, onde constatou-se que a maiores descargas ocorridas na bacia estavam relacionadas com os maiores índices pluviométricos, concordando com Carvalho (2000b), o qual cita que, para muitos cursos d’água, o período chuvoso representa uma média de 70 a $90 \%$ na quantidade de carga sólida transportada em todo o ano hidrológico.

É importante mencionar ainda que os dados coletados pela ANA possuem inúmeras limitações, principalmente em relação à frequência das coletas ao longo dos anos.

Para a maioria dos anos avaliados foram registradas somente duas coletas, uma no período seco e outra no período chuvoso.

Há ainda casos, como o ano de 2011 em que só foram coletados dados para um período sazonal. Tais fatos comprometem um pouco os resultados, sobretudo quando se pretende estimar a descarga sólida de uma bacia. Rodrigues et al. (2013) citam que a produção de informações qualitativas e quantitativas consistentes que possam descrever com precisão os processos é um dos maiores desafios nos estudos hidrossedimentológicos.

Entretanto, essa é a realidade observada em relação ao monitoramento para maioria das estações existentes no país.

\section{Produção de Sedimentos na Bacia do Rio do Coco}

Os resultados obtidos para a produção de sedimentos em suspensão nas principais subbacias da bacia do Rio do Coco entre os anos de 2015 e 2016 estão demonstrados na tabela 3.

Como pode ser visualizado na tabela 3 a 
produção de sedimentos em suspensão nas subbacias analisadas é influenciada diretamente pela sazonalidade. Nos meses de junho de 2015, dezembro, fevereiro e abril de 2016 foram observados os maiores valores na produção de sedimentos em todas as sub-bacias, o que já era esperado. Nestes meses, provavelmente, houve um maior desprendimento de partículas sólidas em função do aumento do escoamento superficial ocorrido no período.

Tabela 3 - Produção de sedimentos em suspensão nas sub-bacias principais da bacia do Rio do Coco.

\begin{tabular}{|c|c|c|c|c|c|}
\hline \multirow{4}{*}{ Meses } & \multicolumn{3}{|c|}{ Sub-bacias da Alta Bacia } & \multicolumn{2}{|c|}{ Sub-bacias da Baixa Bacia } \\
\hline & Alto Rio do Coco & Rib. Piedade & Rib. Surubim & Rib. Prata & Baixo Rio do Coco \\
\hline & Pss & Pss & Pss & Pss & Pss \\
\hline & \multicolumn{5}{|c|}{$\left(t .-k m^{-2} \cdot m \hat{e} s^{-1}\right)$} \\
\hline Abr/15 & 0,184 & 0,102 & - & 0,0558 & - \\
\hline Jun/15 & 0,546 & 0,641 & 0,069 & 0,0325 & 0,4535 \\
\hline Ago/15 & 0,003 & 0,036 & $\mathrm{~S}$ & 0,0047 & 0,0153 \\
\hline Out/15 & $\mathrm{S}$ & 0,031 & $\mathrm{~S}$ & $\mathrm{~S}$ & 0,0023 \\
\hline Dez/15 & 0,238 & 0,178 & $\mathrm{~S}$ & $\mathrm{~S}$ & 0,0134 \\
\hline Fev/16 & 0,452 & 0,369 & 0,373 & 0,0993 & 0,4984 \\
\hline Abr/16 & 1,167 & 2,620 & 0,819 & 0,2915 & 0,9403 \\
\hline Anual & 2,592 & 3,980 & 1,262 & 0,4838 & 1,9232 \\
\hline
\end{tabular}

Nota: Pss = produção de sedimentos em suspensão (ton. $\mathrm{km}^{-2}$. mês $\mathrm{s}^{-1}$ ); $\mathrm{S}=$ curso d’água seco.

As sub-bacias da alta bacia do Rio do Coco (Alto Rio do Coco, Ribeirão Piedade e Ribeirão Surubim) produziram mais sedimentos por área em relação às sub-bacias da baixa bacia, com destaque as sub-bacias do Ribeirão Piedade e Alto Rio do Coco, indicando que os conflitos de uso nas APPs podem ter influenciado nos resultados.

A primeira produziu 3,98 t. $\mathrm{km}^{-2} \cdot \mathrm{ano}^{-1}$, enquanto que a segunda produziu 2,59 t. $\mathrm{km}^{-}$ ${ }^{2}$.ano ${ }^{-1}$. Resultados semelhantes foram encontrados por Souza (2011) ao estudar a produção de sedimentos na bacia do Rio Capibaribe, onde observou, para o período chuvoso, valores de 3,42 t. $\mathrm{km}^{-2}$ ano $^{-1}$ em 2009 e 3,75 t. km-2 ano-1 em 2010.

A sub-bacia do Ribeirão Surubim apesar de localizar-se na região da alta bacia produziu 1,26 t. $\mathrm{km}^{-2}$.ano ${ }^{-1}$, o que pode estar relacionado com sua densidade hidrográfica comparada as demais bacias da alta bacia, bem como com suas características de relevo locais. Além disso, para esta bacia foi observada a interrupção do fluxo hídrico do canal principal em alguns meses do período seco.

A sub-bacia do Ribeirão Prata apresentou os resultados de produção mais baixos em relação as demais bacias estudadas, sendo observado um valor de 0,48 t. $\mathrm{km}^{-2}$.ano ${ }^{-1}$ de sedimentos produzidos, o que pode estar associado com os baixos percentuais de conflitos de uso do solo em APP (22,73\%), comparados às demais subbacias e com as características do relevo, refletindo em suaves declives, com presença de poucos canais por área.

Para a sub-bacia do Baixo Rio do Coco observou-se uma descarga de sólidos suspensos de 11.700 ton/ano, porém, a produção de sedimentos suspensos correspondente a área incremental dessa descarga é de somente 1,92 t.km². ano $^{-1}$.

Vale ressaltar que esse ponto é considerado o exutório da grande bacia do Rio do Coco, ou seja, os resultados encontrados indicam a produção de sedimentos em toda a bacia e o que está sendo entregue para a bacia do Rio Araguaia. Esse valor de produção é considerado baixo diante da produção de sedimentos suspensos em outras bacias brasileiras.

Destaca-se ainda que o cronograma das medições, as quais ocorreram bimestralmente, pode ter influenciado nos resultados. Acredita-se que medições mensais poderiam representar melhor a realidade de campo, entretanto, esse processo é muito complexo, o que exige grande logística e recursos financeiros.

Conforme Carvalho (2000a), resultados de produção de sedimentos em suspensão de até 35 t.km ${ }^{-2}$.ano ${ }^{-1}$ ainda são considerados baixos. Para ser considerada uma produção alta a bacia deverá 
produzir 175 t. $\mathrm{km}^{-2}$.ano ${ }^{-1}$.

Feitosa \& Iost (2011) encontraram valores de produção específica de sedimentos em suspensão para as bacias dos Rios Manuel Alves Grande e Manuel Alves Pequeno de 21,94 t. km ${ }^{-2} \cdot \mathrm{ano}^{-1} \mathrm{e}$ 25,05 t. km ${ }^{-2} \cdot \mathrm{ano}^{-1}$, respectivamente. Melo et al. (2008) registraram 456,6 t. $\mathrm{km}^{-2}$ ano $^{-1}$ de produção de sedimentos em suspensão em estudos na microbacia do Riacho Jacu no semiárido pernambucano, valores estes considerados altos.

Os autores atribuíram os resultados como o reflexo das práticas agrícolas empregadas na área da bacia. Resultados moderados de produção de sedimentos foram encontrados por Andrade (2013) ao estudar o Alto Rio São Francisco, onde foi registrado $101,84 \mathrm{t} . \mathrm{km}^{-2}$. ano ${ }^{-1}$ e Rodrigues et al. (2013) em pesquisa realizada em duas microbacias no semiárido sobre manejos diferentes, onde obtiveram valores de produção de 145 e 139 ton t. $\mathrm{km}^{-2}$, respectivamente.

Após somar as produções de todas as subbacias, exceto a da sub-bacia do Baixo Rio do
Coco verifica-se um total de $8,31 \mathrm{t} . \mathrm{km}^{-2}$.ano ${ }^{-1}$ de sedimentos em suspensão sendo produzidos pelas sub-bacias do Alto Rio do Coco, Ribeirão Piedade, Ribeirão Surubim e Ribeirão Prata e lançados no Rio do Coco, porém, esse quantitativo diverge bastante do que está sendo medido no ponto do Baixo Rio do Coco (1,92 t.km ${ }^{-2}$ ano $^{-1}$ ), desse modo, os resultados indicam que aproximadamente $6,39 \mathrm{t} \cdot \mathrm{km}^{-2} \cdot \mathrm{ano}^{-1}$ de sedimentos estão sendo depositados ao longo dos canais de drenagem.

Tal quadro confirma a tendência para acúmulo de sedimentos no Rio do Coco apresentado pelo índice de sinuosidade do canal calculado durante a análise morfométrica da bacia em estudos realizados por Almeida et al. (2016) na referida bacia. Em síntese, os resultados indicam que a alta bacia é responsável por grande parte dos sedimentos produzidos, ocorrendo na baixa bacia a deposição das partículas transportadas. Esse cenário sugere que tal produção é influenciada pelos conflitos de uso em APPs e pelas características físicas da bacia.

\section{CONCLUSÕES}

Os resultados encontrados nesta pesquisa permitem concluir que:

- Os efeitos da sazonalidade interferiram diretamente nos resultados, influenciando os valores de sólidos suspensos totais e vazão, sendo observadas elevações destes parâmetros nos meses de maiores índices pluviométricos;

- A região da alta bacia foi responsável pela maior produção e transporte de sedimentos, com destaque as sub-bacias do Ribeirão Piedade e Alto Rio do Coco que apresentaram concentrações elevadas durante toda a pesquisa, sugerindo relação direta com os conflitos de uso do solo em APPs;

- Os resultados mais representativos em relação à produção e transporte de sedimentos para as subbacias do Ribeirão Piedade e do Alto Rio do Coco apontam uma relação com o sistema de drenagem e com as características de relevo, indicando que os conflitos de uso em APP podem intensificar toda a dinâmica de produção e transporte;

- A região da alta bacia é responsável pelo maior aporte de sedimentos, ocorrendo na baixa bacia grande deposição dos materiais transportados, assim, os sedimentos produzidos pelas sub-bacias da bacia do Rio do Coco não chegam em sua totalidade no Rio Araguaia, provavelmente estes estão sendo depositados ao longo do canal de drenagem;
- As sub-bacias com maiores percentuais de áreas de preservação permanente preservadas (de acordo com os limites estabelecidos por lei) apresentaram menores índices de produção e transporte de sedimentos, sugerindo que a vegetação ripária é de fundamental importância para a manutenção dos corpos hídricos em uma bacia;

- O estudo realizado sinaliza que a produção e transporte de sedimentos na bacia do Rio do Coco tem relação direta com os conflitos de uso existentes em APPs. Entretanto, deve-se considerar ainda, os aspectos físicos de cada subbacia, pois os mesmos podem ser determinantes em todo o processo.

Há de se destacar, portanto, os desafios para a realização deste trabalho, principalmente no que diz respeito ao monitoramento hidrossedimentológico, o qual exigiu planejamento e logística de campo bem elaborados, além de inúmeros equipamentos para avaliar as diversidades dos canais encontrados na bacia. Por fim, sugere-se a realização de estudos relacionados aos aspectos físicos da bacia e a continuidade do monitoramento dos conflitos de uso do solo e do transporte de sedimentos, para a produção de um banco de dados que permita avaliar as variações espaciais e temporais de descarga na bacia do Rio do Coco e suas contribuições para o Rio Araguaia. 


\section{AGRADECIMENTOS}

Os autores agradecem ao Instituto Federal do Tocantins/Campus Paraíso do Tocantins, ao Laboratório de Processamento de Imagens e Geoprocessamento (LAPIG) da Universidade Federal do Goiás e a Universidade Estadual do Tocantins (UNITINS) pelo apoio durante a pesquisa e a CAPES pela bolsa concedida.

\section{REFERÊNCIAS}

ALMEIDA, R.F.B.; BAYER, M.; FERREIRA JÚNIOR, L.G. Compartimentação morfométrica da Bacia do Rio do Coco como subsídio a análise de fragilidade ambiental. Mercator, Fortaleza, v. 15, n. 4, p. 88, out./dez., 2016.

ANA - AGÊNCIA NACIONAL DE ÁGUAS. HidroWeb Sistema de Informações Hidrológicas. Disp. em: http://hidroweb.ana.gov.br/Estacao.asp?Codigo=27110000. Acesso em: 25 de novembro de 2016.

ANA - AGÊNCIA NACIONAL DE ÁGUAS. Sistema de Monitoramento Hidrológico/Série Histórica. Disp. em: http://mapas-hidro.ana.gov.br/Usuario/Exportar.aspx?bac=2\& sub=27\&est=93049580. Acesso em: 25 de novembro de 2016 .

ANDRADE, M.A. Análise da dinâmica hidrossedimentológica na estação de porto das andorinhas no Rio São Francisco, Minas Gerias. In: SIMPÓSIO BRASILEIRO DE RECURSOS HÍDRICOS, XX, Bento Gonçalves, 2013, Anais....Bento Gonçalves, 2013, p. 06.

ANTONELI, V. Dinâmica do uso da terra e a produção de sedimentos em diferentes áreas fontes na Bacia Hidrográfica do Arroio Boa Vista-Guamiranga - PR. Curitiba. 2011. 39 p. Tese (Doutorado em Geografia) Universidade Federal do Paraná.

BRASIL. Lei n ${ }^{\circ} \mathbf{1 2 . 6 5 1}$ de 25 de maio de 2012. Dispõe sobre a proteção da vegetação nativa.

CARVALHO, N. O. Guia de Avaliação de Assoreamento de Reservatórios. Brasília: ANEEL. 2000a. 92 p.

CARVAlHO, N.O. Guia de Práticas Sedimentométricas. Brasília: ANEEL. 2000b. 23 - 59 p.

CARVALHO, N.O. Hidrossedimentologia Prática. 1ed. Rio de Janeiro: CPRM, 372 p., 1994.

CARVALHO, N.O. Hidrossedimentologia prática. 2. ed. Rio de Janeiro: Rio de Janeiro, RJ: Interciência, 2008. 372 p.

COSTA, C.D.O.; ALVES, M.C.; SOUSA, A.P.; SILVA, H.R.; GONZÁLES, A.P.; AVALOS, J.M.M. Produção e deposição de sedimentos em uma sub-bacia hidrográfica com solos suscetíveis à erosão. Irriga, Botucatu, v. 21, n. 2, p. 285, mai./jun., 2016.

FEITOSA, T.B. \& IOST, C. Dinâmica hidrossedimentológica de duas sub-bacias hidrográficas localizadas no Estado do Tocantins. Rev. Acad., Ciênc. Agrár. Ambient., Curitiba, v. 9, n. 2, p. 127 e 128, abr./jun., 2011.

GARRIDO, J.W.A.; SOUSA, T.M.I.; ISMAEL, L.L.; CRISPIM, D.L.; FERREIRA, P.M.L.; QUEIROZ, M.M.F. Transporte de sedimento suspenso na bacia hidrográfica do Rio PiancóPiranhas-Açu. In: ENCONTRO NACIONAL DE ENGENHARIA DE SEDIMENTOS, XI, 2014, João Pessoa. Anais... João Pessoa, 2014. p. 07.

GONZÁLEZ, J.E.; JARAMILLO, L.J.M. Propuesta metodológica para el análisis morfosedimentológico en cuencas altamente urbanizadas. Caso de estudio quebrada Doña María (Colombia). Revista Ingenierías Universidad de Medellín, v. 9, n. 16, p. 15, enero-junio de 2010.

HARTWIG, M.; SCHAFFER, M.; THEURING, P.; AVLYUSH, S.; RODE, M.; BORCHARDT, D. Cause-effect-response chains linking source identification of eroded sediments, loss of aquatic ecosystem integrity and management options in a steppe river catchment (Kharaa, Mongolia). Environ. Earth Sci., v. 75, n. 855, p. $02-03,2016$.
MELO, R.O.; CANTALICE, J.R.B.; ARAÚJO, A.M.; CUNHA FILHO, M. Produção de sedimento suspenso de uma típica bacia hidrográfica semiárida. In: ENCONTRO NACIONAL DE ENGENHARIA DE SEDIMENTOS, VIII, 2008, Campo Grande. Anais... Campo Grande, 2008, p. 11.

MINELA, J.P.G. \& MERTEN, G.H. Monitoramento de bacias hidrográficas para identificar fontes de sedimentos em suspensão. Ciência Rural, Santa Maria, v. 41, n.3, p. 426, mar., 2011.

PANWAR, S.; KHAN, M.Y.A.; CHAKRAPANI, G.J. Grain size characteristics and provenance determination of sediment and dissolved load of Alaknanda River, Garhwal Himalaya, India. Environ. Earth Sci., v. 75, n. 91, p. 04, 2016.

RODRIGUES, J.O.; ANDRADE, E.M.; PALÁCIO, H.A.Q.; MENDONÇA, L.A.R.; SANTOS, J.C.N. dos. Sediment loss in semiarid small watershed due to the land use. Revista Ciência Agronômica, v. 44, n. 3, p. 489 e 495, jul./set., 2013.

RODRIGUES, V.A.; SÁNCHEZ-ROMÁN, R.M.; TARJUELO, J.M.; SARTORI, M.M.P.; CANALES, A.R. Avaliação do Escoamento e interceptação da água das chuvas. Irriga, Botucatu, Ed. Especial, p. 08, 2015.

SANTOS, D. S. \& SPAROVEK, G. Retenção de sedimentos removidos de área de lavoura pela mata ciliar, em Goiatuba (GO). Revista Brasileira de Ciência do Solo, v. 35, n. 5, p. 1814-1817, set/out, 2011.

SILVA, J.L.; TONELLO, K.C.; VALENTE, R.A.; MINGOTI, R. Diagnóstico ambiental como subsídio à restauração florestal e manutenção hidrológica da Bacia do Ribeirão dos Pinheirinhos, Brotas - SP. Irriga, Botucatu, 21, 08, jan./mar., 2016.

SOUZA, W.L.S. Produção de sedimentos da bacia hidrográfica do Rio Capibaribe para zona costeira da região Metropolitana do Recife. Recife 60f. 2011. Dissertação (Mestrado em Ciência do Solo), Universidade Federal Rural de Pernambuco.

STRAHLER, A. N. Hypsometric (area-altitude) analysis and erosional topography. Geological Society of America Bulletin, v. 63, p. 1117 - 1142, 1952.

SUARÉZ, P.A.; VEGA, M.; PARDO, R.; ORFEO, O.; CUESTA, J.L.G.; RONCO, A. Hydrochemical and sedimentological dynamics in a subtropical plain river: assessment by multivariate statistical analysis. Environ. Earth Sci., v. 75, n. 1004, p. 02, 2016.

ZANATA, M.; PISSARRA, T.C.T.; FERRAUDO, A.S.; RANZINI, M.; CAMPOS, S. Effect of soil use on the quality of water resource in watershed using multivariate statistical analysis. Irriga, Botucatu, v. 20, p. 787, out./dez., 2015.

Submetido em 9 de julho de 2019 Aceito para publicação em 5 de outubro de 2020 